\title{
When a Case of Ulcerative Colitis Requires Mucosectomy and Hand-Sewn Ileo-Anal Pouch Anastomosis
}

\author{
Mary Teresa M O'Donnell ${ }^{1,2^{*}}$, Joshua IS Bleier ${ }^{3}$ and Gary Wind ${ }^{4}$ \\ ${ }^{1}$ Colon \& Rectal Surgery Fellow, University of Pennsylvania, USA \\ ${ }^{2}$ Assistant Professor of Surgery, Uniformed Services University of the Health Sciences, USA \\ ${ }^{3}$ Associate Professor of Surgery, Department of Surgery, University of Pennsylvania, USA \\ ${ }^{4}$ Professor of Surgery, Uniformed Services University of the Health Sciences, USA
}

*Corresponding author: Mary Teresa M O'Donnell, Colon \& Rectal Surgery Fellow, ACS AEI Educational Fellow, University of Pennsylvania, USA; Assistant Professor of Surgery, Uniformed Services University of the Health Sciences, USA, E-mail: maryteresaodonnell@gmail.com

\section{Summary}

Treatment of ulcerative colitis with total proctocolectomy and ileal pouch anal anastomosis (IPAA) provides a near cure for the bowel component of ulcerative colitis, a restoration of bowel continuity, and the possibility of normal defecation behaviors for patients. The majority of these procedures are carried out using endto-end circular stapling devices, which shorten surgery times and have been shown to give better long-term continence, when compared to mucosectomy and/ or hand-sewn pouch-anal anastomosis [1]. There have been no long-term data to show any oncologic advantage to mucosectomy and the functional advantages of the double stapled approach far outweigh any reputed benefit of mucosectomy [2]. Although advanced age is a relative contraindication to IPAA, most Colon and Rectal surgeons will perform IPAA in patients who demonstrate good fecal continence and agree to postoperative anastomotic surveillance via pouchoscopy. These patients continue to need surveillance of the remaining pouch and mucosal cuff for signs of inflammation, dysplasia, or adenomatous change since there remains a small risk of persistent disease at the remaining native rectal mucosa. While IPAA with mucosectomy can theoretically eliminate this risk, recurrent dysplasia and malignancy can still occur since there remains a risk of a residual mucosal component $[1,3]$. Residual nests of rectal mucosa have been found in up to $21 \%$ of patients who have undergone mucosectomy [4]. In addition, it is associated with increased risk of incontinence and operative difficulty. As a result, mucosectomy is rarely performed.

Nevertheless, there are cases where mucosectomy and hand-sewn ileal anal anastomosis may be required. If there are significant mucosal changes associated with ulcerative colitis that persist low in the rectum to the dentate line, the double-stapled anastomosis may be inadequate. In addition, patients with prior benign anal canal disease or a history of anal surgery may be unsuitable for double stapled anastomosis. These are the patients IPAA with mucosectomy may be required despite the risk of poorer function. We present a case of a male who underwent completion proctectomy with mucosectomy, hand-sewn ileal pouch anal anastomosis and diverting loop ileostomy for refractory colitis with good success.

\section{Case Presentation}

A 52-year-old male presented to our clinic for restorative proctectomy and ileal-pouch anal anastomosis (IPAA). The patient had undergone urgent laparoscopic subtotal colectomy and right nephrectomy for fulminant colitis and a synchronous right renal cancer. Final surgical pathology confirmed ulcerative colitis and he recovered uneventfully. He returned after resolution of acute disease, weaning of steroids, and normalization of nutritional parameters desiring return of continence

Citation: O'Donnell MTM, Bleier JIS, Wind G (2018) When a Case of Ulcerative Colitis Requires Mucosectomy and Hand-Sewn Ileo-Anal Pouch Anastomosis. Int J Surg Res Pract 5:079. doi. org/10.23937/2378-3397/1410079

Accepted: June 05, 2018; Published: June 07, 2018

Copyright: @ 2018 O'Donnell MTM, et al. This is an open-access article distributed under the terms of the Creative Commons Attribution License, which permits unrestricted use, distribution, and reproduction in any medium, provided the original author and source are credited. 


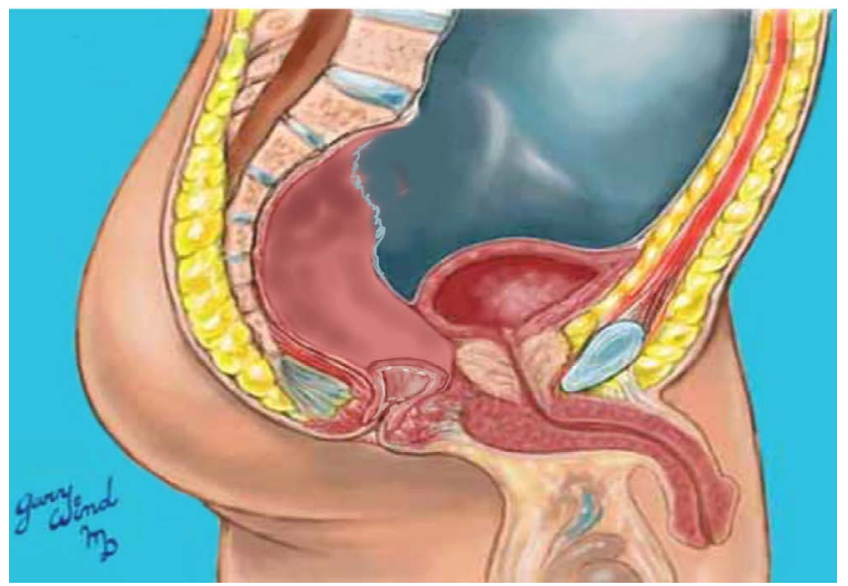

Figure 1: Proctectomy to level just proximal to the dentate line.

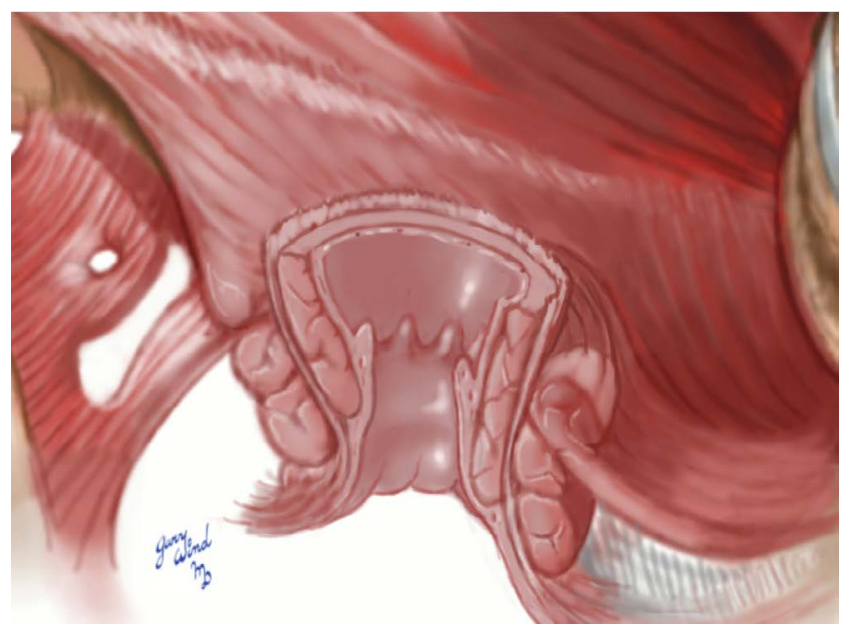

Figure 2: Anal canal after proctectomy.

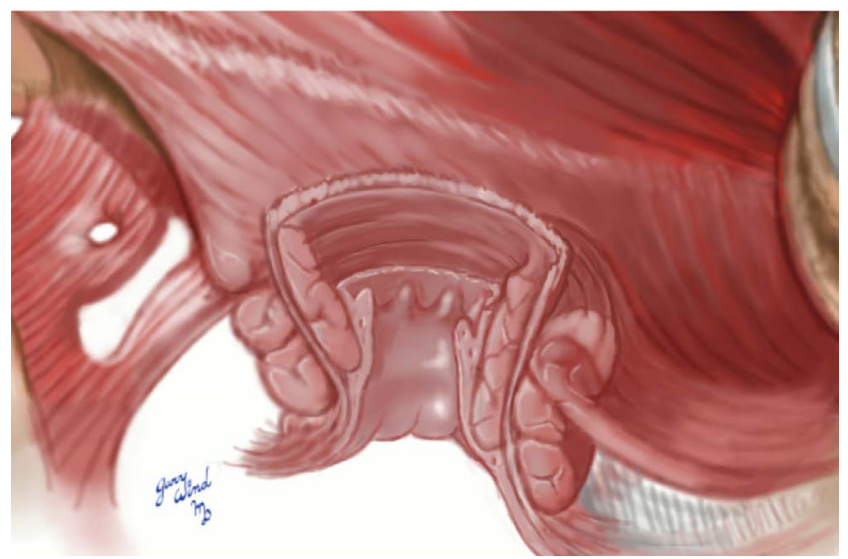

Figure 3: Anal canal after mucosectomy.

through IPAA. The patient had been evaluated to rule out any indication of Crohn's by gastroenterology. He had a history of surgical interventions for hemorrhoidal disease but had always demonstrated normal fecal continence. Digital rectal examination and anoscopic examination revealed moderate anal canal stenosis and severe mucosal irregularity. In the operating room, proctectomy was completed without difficulty (Figure 1), but as expected, a double stapled anastomosis was unable to be performed due to the mucosal irregularity. This was confirmed by the inability to pass the smallest rectal sizer. The rectum

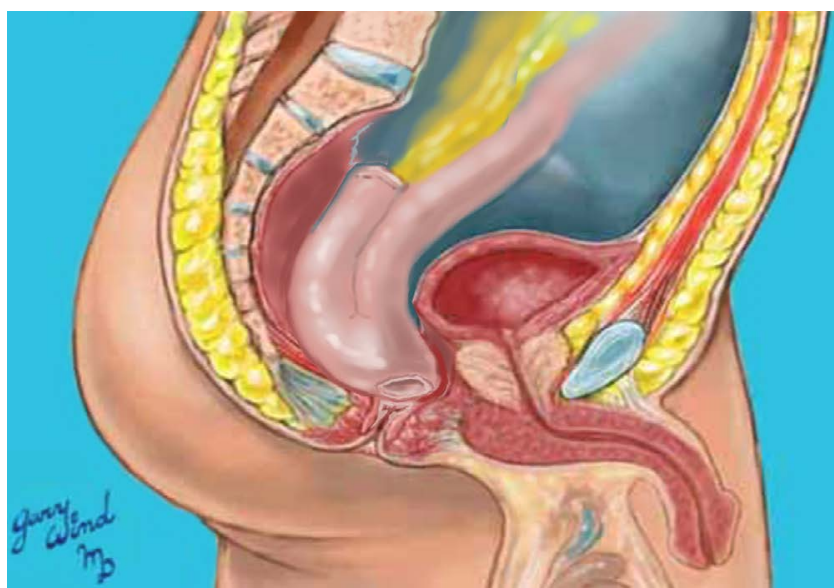

Figure 4: Ileo-anal pouch anastomosis after mucosectomy.

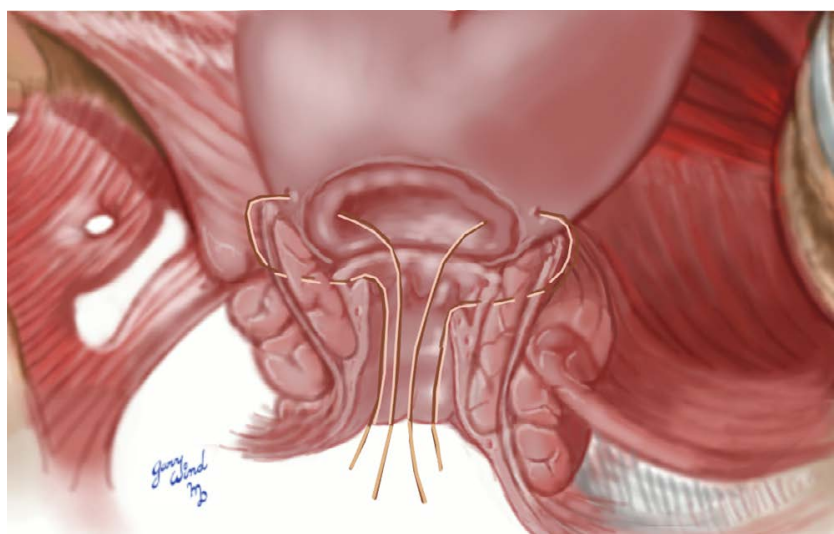

Figure 5: Full-thickness interrupted sutures of the circumferential hand-sewn IPAA.

was transected at the level of the levators and mucosectomy of the anal canal was performed (Figure 2, Figure 3 and Figure 4). The pouch was advanced through the anal aperture to ensure adequate length and mobility prior to performing a tension-free hand-sewn anastomosis (Figure 5) with the aid of a lone-star retractor. This was performed without complication, a loop ileostomy performed for fecal diversion, and the patient recovered uneventfully. Two months after surgery, he was evaluated with GGE demonstrating good healing of the pouch and anastomosis as well as the ability to functionally retain the contrast. His DRE reveals mild stenosis, but easily dilated with the examining finger. He underwent uncomplicated ileostomy closure with excellent functional results.

\section{Quick Hit Learning Points}

1. Mucosectomy is associated with decreased functional results and no improved oncologic results.

2. Good preoperative continence should be assessed prior to performing IPAA and counseling regarding the risk of impaired fecal incontinence should be performed prior to consent.

3. Preoperative examination prior to IPAA formation should include a digital rectal exam with anoscopy or proctoscopy in patient's ulcerative colitis in order to ensure normal anatomy. 


\section{Senior Author Commentary}

When performing restorative proctocolectomy with a plan for double-staple J-pouch-anal anastomosis, appropriate preoperative consideration of potential issues can help mitigate unforeseen complications in the operating room. In the pre-operative history, it is critical to document normal continence to ensure that a patient will be a reasonable candidate for J-pouch reconstruction. A history of disturbed continence or significant urgency with liquid stool may portend an unacceptable functional outcome. Similarly, if a double-stapled anastomosis is anticipated, normal distal rectal and anal canal anatomy is required, and so a careful anoscopic and digital rectal examination is critically important.

Intra-operatively, there are well-known key maneuvers that are required to maximize mobilization of the ileal mesentery to ensure adequate reach for pouch anastomosis. These maneuvers include resection of the ileocolic pedicle, full mobilization of ileal mesentery to the level of the anterior duodenum, as well as fenestration of the peritoneal lining of the mesentery. Being able to get the apex of the proposed pouch to reach the pubic symphysis is a reliable proxy for adequate length. When it becomes necessary to perform a hand-sewn pouch-anal anastomosis, it is important to realize the need for significantly more length for the pouch to reach, since the anal canal length may vary from as short as $4-5 \mathrm{~cm}$ to as long as $8-10 \mathrm{~cm}$ in some males. Thus, careful attention to maximization of lengthening procedures is required. A two-team simultaneous approach is almost always needed to ensure that the pouch can reach, and assistance in pouch stabilization during anastomosis will help maximize success. It is important to realize, that there will almost always be some degree of tension on the mesenteric pedicle after pouch creation and anastomosis; one must always be able to palpate a pulse in the pouch mesentery, even in the face of some tension. Pouch surgery is technically demanding, and experience plays a large role in success, but in any case, careful attention to appropriate patient selection and key operative maneuvers will maximize the chance for success.

\section{References}

1. Remzi F, Church JM, Bast J, Lavery IC, Strong SA, et al. (2001) Mucosectomy vs. stapled ileal pouch-anal anastomosis in patients with familial adenomatous polyposis: functional outcome and neoplasia control. Dis Colon Rectum 44: 1590-1596.

2. Church $J(2005)$ lleoanal pouch neoplasia in familial adenomatous polyposis: an underestimated threat. Dis Colon Rectum 48: 1708-1713.

3. Ooi BS, Remzi FH, Gramlich T, Church JM, Preen M, et al. (2003) Anal transitional zone cancer after restorative proctocolectomy and ileoanal anastomosis in familial adenomatous polyposis: report of two cases. Dis Colon Rectum 46: 1418-1423.

4. O'Connell PR, Pemberton JH, Weiland LH, Beart Jr RW, Dozois RR, et al. (1987) Does rectal mucosa regenerate after ileoanal anastomosis? Dis Colon Rectum 30: 1-5. 